\title{
Der raumwirtschaftliche Ansatz: der Aufstieg einer steuerungsorientierten, normativ geprägten Expertenkultur
}

\section{Christoph Scheuplein}

Westfälische Hochschule, Institut Arbeit und Technik, Munscheidstraße 14, 45886 Gelsenkirchen, Germany

Correspondence to: Christoph Scheuplein (scheuplein@iat.eu)

Received: 30 November 2016 - Revised: 23 February 2017 - Accepted: 2 April 2017 - Published: 25 April 2017

Kurzfassung. In today's corpus of economic geography textbooks published in the German-speaking countries, the spatial science approach is described as involving a neoclassical view of economics, a chorological explanatory strategy, (neo)positivist roots, and a focus on developments that have occurred since 1969. This approach has received sweeping criticism for its "undersocialization", its "spatial fetishism", and its exclusively quantitative methodology. The article proposes to abandon the dominant historiography of science, which focuses on national economies and takes a positivist perspective, in favor of a form of contextualization that has its roots in the sociology of knowledge.

The article examines relevant publications in journals and textbooks to show that what has been known as the quantitative revolution did not become widely accepted among German-speaking researchers until the late 1970s, long after it originally began to emerge. This process was characterized by a shift toward a consultative perspective that researchers adopted in hopes of becoming involved in the spatial planning initiatives of the state. In the resulting usage context of the Keynesian interventionist state, a culture of economic geography experts developed that discussed methods and concepts in an innovative and pluralist manner.

\section{Einleitung}

In der gegenwärtigen deutschsprachigen wirtschaftsgeographischen Diskussion ist der raumwirtschaftliche Ansatz (RA) ein gemeinsamer Fixpunkt. Sowohl seine Vertreter wie auch seine Kritiker, z.B. aus dem Lager der institutionalistisch oder evolutionär argumentierenden Wirtschaftsgeographie, sehen ihn als eine Theorie mit klar umrissenen Annahmen, Aussagen und Grenzen. Ebenfalls ist man sich weitgehend darin einig, dass dieser Ansatz in einem kurzen Zeitraum etabliert wurde und zu einem vollständigen Umbruch in der wissenschaftlichen Diskussion geführt hat. Konsensfähig ist hier sicher die pointierte Feststellung von Haas und Neumair (2007:4): ,In den 1960er Jahren kommt es zur bewussten Abkehr von der individualistischen Länderkunde und zu einer abstrakten, systematischen und theoretisch fundierten wirtschaftsgeographischen Forschung. Diesen Paradigmenwechsel hin zur Wirtschaftsgeographie als Raumwirtschaftslehre, der in Deutschland vor allem durch den Kie- ler Geographentag 1969 markiert wird, bezeichnet man auch als quantitative Revolution." Durch diese symbolhafte Kopplung mit einem wissenschaftlichen Kongress in diesem Zitat wie im verbreiteten theoriegeschichtlichen Gedächtnis wird die Schnelligkeit und Vollständigkeit des Wechsels unterstrichen (vgl. Weichhart, 2004:15; Kulke, 2008:14 und Braun und Schulz, 2012:12 f.). Zugleich werden dem RA bereits für die Zeit ab 1969 klare Konturen zugesprochen (,,abstrakt, systematisch, theoretisch") und er wird mit der Quantitativen Revolution gleichgesetzt. All dies hat dazu beigetragen, dass er auch in der heutigen Theoriediskussion als eine Negativfolie genutzt wird, die bei der Schärfung des eigenen Profils hilft. Entsprechend offensiv wird mit Gegensatzpaaren wie z.B. atomistische vs. eingebettete Akteure, vollständige vs. unvollständige Information, Raum als Kausalfaktor vs. Raum als Perspektive gearbeitet.

Dabei ist den Autoren selbstverständlich bewusst, dass wesentliche Theorieteile des RA - man denke an die Beiträge, die von Thünen, Weber, Christaller und Lösch erarbeitet 
haben, - zeitlich, theoriestrategisch und disziplinär weit entfernt von dem entstanden, was dann in den 1960er Jahren als „Spatial Analysis“ benannt wurde (vgl. Bathelt und Glückler, 2012:37). Diese Entstehungskontexte bzw. die Vermittlungsschritte bis zu dem uns heute als ,raumwirtschaftlicher Ansatz" bekannten Theoriekorpus werden jedoch weitgehend vernachlässigt unter der Annahme, dass Theoriegehalte erst in dem Augenblick Relevanz erlangten, in dem sie von Universitätsprofessoren an einer deutschsprachigen Universität im Fach Geographie rezipiert wurden. In diesem Sinne ist die heute dominierende Theoriegeschichtsschreibung in der Wirtschaftsgeographie doppelt beschränkt: zum einen stellt sie nur eine Universitäts- bzw. Disziplingeschichte dar, zum anderen blickt sie nur auf den deutschen Sprachraum.

In diesem Beitrag wird dafür plädiert, diese national zentrierte Universitätsgeschichte nicht fortzusetzen, sondern den Weg zu einer disziplin- und länderübergreifenden Theoriegeschichte zu beschreiten. (Wobei an dieser Stelle nur ein knapper Ausblick auf die englischsprachigen Theoriediskussionen geleistet werden kann.) Der disziplinäre Sonderweg der (deutschsprachigen) Humangeographie, die sich im 20. Jahrhundert jenseits aller sozialwissenschaftlichen Theoriebildung konstituiert hatte, wurde mit dem Kieler Geographentag nicht verlassen (Michel, 2014; Arnreiter und Weichhart, 1998:60; Weichhart, 2016), sondern als eine - verspätete - disziplinäre Modernisierung fortgesetzt (Blotevogel, 2003:7 f.). Insofern bleibt die heutige Fixierung auf „Kiel“ und das damit verbundene Konstrukt eines ,raumwirtschaftlichen Ansatzes“ ein Erbe dieses Sonderwegs. In diesem Beitrag sollen dagegen die Elemente des RA im theoriegeschichtlichen Kontext rekonstruiert und gefragt werden, was das eigentlich Neue und für die Wirtschaftsgeographie Verdienstvolle an diesem Ansatz darstellte.

Im Folgenden wird zunächst der hier gewählte methodische Ansatz vorgestellt (Abschnitt 2) und danach die gegenwärtig vorherrschende Charakterisierung des RA als Raumdeterminismus, Untersozialisierung Quantifizierung skizziert (Abschnitt 3). Anschließend wird anhand von zeitgenössischen Überblicksartikeln zur Fachentwicklung sowie mit einer Themenanalyse von Artikeln der ,Zeitschrift für Wirtschaftsgeographie" und von akademischen Lehrbüchern gezeigt, dass der RA sich im deutschsprachigen Raum erst ab dem Ende der 1970er Jahre durchsetzte (Abschnitt 4). Dabei war er jedoch weniger durch die ihm heute zugeschriebenen Merkmale gekennzeichnet (Abschnitt 5). Entscheidend für den innerdisziplinären Erfolg des RA war vielmehr der von ihm propagierte Wechsel der Adressatenperspektive auf raumbezogene Planungsbedarfe in Politik und Verwaltung (Abschnitt 6). Damit war er Teil des gesellschaftlichen Wandels in der Nachkriegsgesellschaft und speziell des keynesianischen Interventionsstaates, für den er wirtschaftsgeographisches Wissen bereitstellte. Im Anschluss daran wird der spezifische „Praxiszugang“ des RA näher betrachtet (Abschnitt 7) und es werden einige Essentials zur Einordnung des RA innerhalb der Subdisziplin Wirtschaftsgeographie festgehalten (Abschnitt 8).

\section{Theoriegeschichtliche Kontextualisierung}

In den vergangenen 15 Jahren hat in der Humangeographie eine Abkehr von einer Theoriedarstellung stattgefunden, in denen jeweils der letzte Stand des positiven Wissens präsentiert wird. So liegen für die Sozialgeographie inzwischen Überblicksdarstellungen vor, in denen Konzepte und konkurrierende Schulen im Kontext zeitgenössischer Problemstellungen und intellektueller Strömungen porträtiert werden (Peet, 1998; Cresswell, 2013; Cox, 2014). In der Wirtschaftsgeographie wurde diese Form inzwischen mehrfach für die Darstellung der anglo-amerikanischen Spatial Analysis gewählt (Gregory, 1981; Barnes, 1998, 2003) bzw. für kurze Überblicksartikel über die Fachgeschichte (Scott, 2000). Im deutschsprachigen Raum gibt es eine Tradition ideologiekritischer Arbeiten zu einer Kontextualisierung der geographischen Wissensentwicklung (Hard, 1979; Schultz, 1980; Eisel, 1980). Daran haben einige Kritiken zur (neoklassischen) Standort- und Raumwirtschaftstheorie angeschlossen, die die Funktionalität der quantitativen Methoden und Modellbildung für gesellschaftliche neue Verwendungskontexte in der staatlichen Raumplanung hervorheben (Läpple, 1991:170188; Krumbein et al., 2008:28 f.; von Frieling, 2008). Direkt auf den RA bezieht sich Schamp (2007), wobei er den Begriff des Denkstils verwendet, der als Spannungsfeld aus staatlichen Vorgaben, Marktchancen und universitären Entscheidungen konzipiert ist. Schamp erkennt für den RA daher seit dem Ende der 1970er eine Hegemonie in Deutschland, wobei dieser sich aufgrund seines marktkonformen Theorieansatzes, aber auch durch seine Beratungskompetenz in der staatlichen Planung und durch seine wissenschaftsinterne Nachwuchspolitik durchsetzen konnte (Schamp, 2007:243 f.). Diese theoriehistorische Herausforderung ist jedoch in der Lehrbuch-Literatur noch nicht aufgenommen worden. Wo Theorieansätze auf der Basis ihrer eigenen Fragestellungen berücksichtigt werden (Bathelt und Glücker, 2012), wird ihre Einordnung und Kritik in einem logischen Raum vorgenommen. In den meisten Fällen wird eine historische Einordnung von Theorien dagegen ausgespart oder in den Vorspann verwiesen (vgl. Kulke, 2008:11-16).

Als Mittel für eine theoriegeschichtliche Rekonstruktion werden in der angelsächsischen Debatte häufig postkonstruktivistische Ansätze angewandt (wegweisend: Gregory, 1994; Barnes, 1994, 1996). Mit ihnen wird die Diskussion allerdings weitgehend auf das Feld der Wissenschaftstheorie verlagert und davon ausgegangen, dass die Forschungs- oder Denkstile ihre eigenen epistemologischen Bewertungsmaßstäbe hervorbringen (Moulines, 2008:122 ff.). StandardmäBig wird dann argumentiert, dass die Universalität, Objektivität und Präzision der quantitativen Methoden bzw. der mathematischen Sprache nur scheinbar bestehe und der Sinngehalt 
des kulturell geprägten und lokal verteilten Wissens nicht erfasst werde (Barnes, 2001, 2006). Die damit häufig verbundene Ablehnung jeder Quantifizierung bzw. Modellbildung trifft dann allerdings nicht nur die Spatial Analysis, sondern jeden Versuch in der Wirtschaftsgeographie, Verhältnisse der Produktion und Distribution von Gütern auch quantitativ zu erfassen (Sheppard, 2001, 2014).

Aussichtsreicher für eine theoriegeschichtliche Darstellung erscheint ein wissenssoziologisches Vorgehen, bei dem die zentralen Gegenstände und Ideen eines wissenschaftlichen Ansatzes im Kontext ihres Entstehungszusammenhanges thematisiert werden. Das Wissen über gesellschaftliche Arenen, in denen wissenschaftliche Fragen oder Lösungen angeregt wurden, über die Akteure, die sich diesen Fragen gestellt haben, und über die Wirkungen, zu denen diese wissenschaftlichen Innovationen unmittelbar geführt haben, stellen einen Erkenntnismehrwert dar, der eine rein systematische Darstellung ergänzt (vgl. Knoblauch, 2005:242). Da die Genese von Theorien und ihre weitere Geltung in den Sozialwissenschaften nie vollständig voneinander getrennt werden können, informiert eine wissenssoziologische Betrachtung auch über potenzielle Verwendungskontexte der Theorie. Demgegenüber stellt die Abstraktion von diesen Kontexten, wie sie in der Standard-Geschichtsschreibung von humangeographischen Theorien vorherrscht, selbst eine konstruktive Leistung dar, die sichtbar zu machen ist. Dies soll im Folgenden angestrebt werden: Was war die gesellschaftliche Konstellation, die den RA hervorgebracht, und welche Strukturierung seiner Wissensbestände folgte daraus?

\section{Der raumwirtschaftliche Ansatz in der Sicht der Anderen}

Der Terminus „raumwirtschaftliche Ansatz“ wurde im deutschsprachigen Raum von Ludwig Schätzl in seiner GieBener Antrittsvorlesung (Schätzl, 1974) und in der ersten Auflage seines wirtschaftsgeographischen Lehrbuchs (Schätzl, 1978) geprägt. Sicher gab es in den 1970er Jahren eine Reihe weiterer neuberufener Professoren auf Lehrstühlen der Wirtschaftsgeographie (teils zusammen mit der Sozialgeographie), die sich ebenso epistemologisch und methodisch an der „Spatial Analysis“ orientierten, z.B. Ernst Giese (berufen 1973), Dietrich Bartels (1973), Gerhard Bahrenberg (1974), Wolf Gaebe (1978) oder Hans-Dieter Haas (1979). Dennoch weist der Entwurf von Schätzl (a) eine besondere inhaltliche Konsistenz auf (die weiter unten im fünften Abschnitt expliziert wird) und führte (b) zu einer wissenschaftlichen Schulbildung, d.h. verfügte über Projekte, Lehrbücher, bevorzugte Publikationsorgane und Veranstaltungen und reproduzierte sich über Qualifikationsarbeiten und Berufungen. Aus diesem Grund wird der RA weiterhin vor allem mit Ludwig Schätzl und den an der TU Hannover habilitierten Fachvertretern (Elmar Kulke, Rolf Sternberg, Knut
Koschatzky, Javier Revilla Diez, Ingo Liefner, Matthias Kiese, Daniel Schiller, Tom Brökel) identifiziert.

Für den so eingegrenzten RA soll in einem ersten Schritt die heute dominierende Vorstellung in der deutschsprachigen Wirtschaftsgeographie ermittelt werden. Um diesen „,kleinsten gemeinsamen Nenner“ in der ,scientific community“ zu rekonstruieren, wird im Folgenden auf bekannte Einführungen in die Teildisziplin Wirtschaftsgeographie zurückgegriffen, soweit sie nicht von den oben genannten Vertretern des RA stammen.

Es können vor allem drei wiederkehrende Elemente festgehalten werden, mit denen gegenwärtig eine raumwirtschaftstheoretische Position umrissen wird. Erstens wird der RA durch seine kausale Einführung der physischen Lagedistanzen bzw. ihrer ökonomischen Fassung als Transportkosten oder Raumüberwindungskosten charakterisiert. Demnach vertritt er durch das Beharren auf den Raum als dem zentralen determinierenden Faktor ein chorologisches Verständnis (Bathelt und Glückler, 2012:37; Braun und Schulz, 2012:13, 27 ff.; Haas und Neumair, 2007:10). Dies wird inhaltlich vor allem durch die einzel- und gesamtwirtschaftlichen Standorttheorien zur Landnutzung, zur Industrie und zu Dienstleistungen repräsentiert (Christaller-Weber-LöschModelle). Zweitens wird diese Erklärungsstrategie innerhalb eines neoklassischen Wirtschaftsmodell artikuliert, z.B. mit Annahmen zum atomistischen, rationalen und informierten Akteur, zur Vollkommenheit der Marktstruktur und zur Homogenität des Raumes (Bathelt und Glückler, 2012:37 f., 113 f.; Braun und Schulz, 2012:13; vgl. auch Maier und Tödtling, 2006:9ff, 24 f.; Haas und Neumair, 2007:4). Drittens wird dieses modelltheoretische Denken wissenschaftstheoretisch im (Neo-)Positivismus bzw. im kritischen Rationalismus fundiert, wobei Bartels (1968a) methodologischer Entwurf als Kronzeuge dient. In der praktischen Forschung werden diesem Programm quantitativ-mathematische Methoden, der methodologische Individualismus und die Methode der isolierenden Abstraktion zugeordnet (Maier und Tödtling, 2006:24 f.). In der angelsächsischen Diskussion stehen diese Veränderungen in der Methodologie und die damit verknüpften neuen Methoden bei der Schilderung des wissenschaftlichen Umbruchs ganz im Vordergrund (vgl. Cresswell, 2013:79-102), weshalb dort auch früher und seitdem intensiver der Begriff der „Quantitativen Revolution“ verwendet wurde (Burton, 1963).

Insgesamt wird der RA als ein recht geschlossenes Theoriegebäude dargestellt, mit einer starken Kongruenz zwischen Methodologie, methodischem Instrumentarium und Kernaussagen. Eine gewisse Rigidität des Ansatzes wird dabei nicht nur von seinen Befürwortern, sondern auch von seinen Kritikern herausgestellt und auf seine Abstammung aus der volkswirtschaftlichen Regionalökonomie bzw. der in den USA disziplinär eigenständigen Regional Science (oder: Spatial Analysis) verwiesen.

Dieses knapp gefasste, aber wohl auch weithin geteilte Grundverständnis des RA soll nun als Suchraster dienen, mit 
dem im nächsten Schritt untersucht werden soll, ab wann und in welchem Maße sich dieses Verständnis in der deutschsprachigen Wirtschaftsgeographie identifizieren lässt.

\section{Der Themen- und Methodenwechsel in den 1970er Jahren}

Der oben zitierten Darstellung eines schnellen und durchgreifenden Umbruchs zum RA können nun erstens andere zeitliche Einordnungen gegenüber gestellt werden: So setzt etwa Schamp (2007:244) dessen Siegeszug erst für das Ende der siebziger Jahre an. Ebenso kann man auf zeitgenössische Einschätzungen aus der Sicht quantitativ-mathematisch orientierter (Wirtschafts-)Geographen verweisen, die in ihren Berichten zum Forschungsstand zu ernüchternden bis pessimistischen Einschätzungen kamen. Kilchenmann (1975:1) diagnostizierte für die deutsche Geographie den Nachholbedarf eines Entwicklungslandes. Lichtenberger (1978:16) kann nur Inseln mathematisch-quantitativer Arbeit an einigen eher peripheren universitären Standorten erkennen. Giese (1980:259) berichtet von einer ,,stark zeitverzögerten Entwicklung“ der quantitativen Geographie, die sich auch bis zum Ende der 1970er Jahre nicht durchsetzen konnte, und Bahrenberg (1979:60) sieht die Entwicklung ,auf halbem Wege zum Stillstand gekommen" (vgl. auch die weiteren Beiträge in Sedlacek, 1979 sowie Hard und Fleige, 1978 zum Zitierverhalten in der Geographie).

Charakteristischerweise blieben die methodologischen Grundprinzipien Bartels (1968a und b) in der Wirtschaftsgeographie umkämpft, was unter anderem zu dem einflussreichen Gegenentwurf einer „Allgemeinen Kulturgeographie“ durch Eugen Wirth führte. Diese sollte eine Suche nach sozialen Regelhaftigkeiten betreiben, solange diese das konzeptionelle Ordnungsprinzip der Landschaft nicht in Frage stellten (Wirth, 1969:186, 1978, 1979). Diese „konservative Umarmung“ (Bartels, 1980) blieb der Gegenstand scharfer Kontroversen (vgl. zusammenfassend Bahrenberg, 1995, 1996; Belina et al., 2009:50 f.).

Dieser Befund eines langsamen Wechsels in den 1970ern soll hier in einem zweiten Schritt anhand der ,Zeitschrift für Wirtschaftsgeographie“, die sich ganz auf die Subdisziplin Wirtschaftsgeographie konzentriert, nachgeprüft werden. Sie war seit ihrem Erscheinungsbeginn im Jahr 1957 Länderkunde-konform aufgestellt und publizierte (neben den Hauptbeiträgen) unter den Rubriken „Bevölkerung“, „Projekte“, „Wirtschaftsgüter“ und „Verkehr“ faktensammelnde Kurzberichte. Im Zeitraum von 1969 bis 1975 brachte sie zunächst nur einzelne theorie- und methodenorientierte Artikel, z.B. zu Regionalisierungsmodellen (Bartels, 1970; Gaebe, 1970), zur Zentralen-Orte-Theorie (Morikawa, 1971) oder zu Beziehungen zwischen Betriebsgröße und Betriebstyp (Haas, 1971) ${ }^{1}$. Unter dieser thematischen Ausdehnung

\footnotetext{
${ }^{1}$ Weitere Beiträge behandelten die Verhaltenstheorien in der Industriegeographie (Krumme, 1972), die Einzelhandelsforschung
}

stechen vor allem die Felder Raumordnung und Regionalpolitik heraus. Während sich in der, Zeitschrift für Wirtschaftsgeographie" zu diesem Themenkreis in den 1960er Jahren nur Kurzberichte sowie eine Artikelfolge finden (beginnend mit Siebert, 1963), tauchen nun die föderale und kommunale Neuordnung, die Gebietsabgrenzung räumlicher Leitbilder und die regionale Strukturpolitik als Gegenstand auf (Bartels, 1970; Gaebe, 1970, [o.V.], 1971; Winkler, 1975; Beck, 1975; Elsasser, 1975). Damit wurde der geographische Schwerpunkt von den überseeischen Entwicklungs- und Schwellenländern, die typischerweise in einer länderkundlichen Qualifikationsarbeit gewählt wurden, in Richtung auf Deutschland, Österreich und der Schweiz verlagert, wenn auch nur in sehr bescheidenem Maße. Auf dem Geographentag in Erlangen und Nürnberg 1971 widmete sich immerhin bereits eine gesamte Sitzung der Verwaltungsgebietsreform in der Bundesrepublik Deutschland (Schöller und Liedtke, 1972).

Insgesamt können aber nur 10 Artikel im Zeitraum 1969 bis 1974 in der „Zeitschrift für Wirtschaftsgeographie“ dem oben skizzierten neuen Grundverständnis zugeordnet werden (Tabelle 1). Erst ab dem Jahr 1975, als immerhin 5 Artikel der neuen Richtung zugeordnet werden können, verstärkte sich dieser Einfluss und blieb dann bis zum Jahr 1980 auf diesem Niveau. Ihr Anteil lag in der zweiten Hälfte der 1970er Jahre in der „Zeitschrift für Wirtschaftsgeographie“, die jährlich durchschnittlich 40 Artikel publizierte (plus diverse Kurzberichte in den oben genannten Kategorien), bei weniger als einem Sechstel.

Als dritter Beleg für einen verzögerten Themen- und Methodenwandel können einschlägige wirtschaftsgeographische akademische Lehrbücher der 1970er Jahre herangezogen werden, in denen der idiographisch-länderkundliche Ansatz bestehen blieb (Voppel, 1970; Otremba, 1978; Wagner, 1981). Dies spiegelte sich auch in schulischen Lehrbüchern (z.B. Nickel und Piepenstock, 1972; Wenzler, 1972; Reidel et al., 1973; Dorner, 1975), die qua Anbindung an kultusministerielle Vorgaben ohnehin zeitversetzt agierten.

Die oben skizzierten drei Elemente - die Transportkostenorientierung bzw. das chorologische Raumverständnis, eine rationale und atomistische Handlungstheorie und eine neopositivistische Methodologie haben sich nur stellenweise und mit vielen Widerständen in der deutschsprachigen Wirtschaftsgeographie der 1970er Jahre durchgesetzt. Für eine systematische Theoriegeschichte ist somit der Bezug auf andere Teildisziplinen bzw. Diskussionen notwendig. Erstens muss man sich bei der Transportkostenorientierung auf eine seit dem 19. Jhd. entwickelte Abfolge von Einzeltheorien beziehen, die dann seit den 1940er Jahren in der volkswirtschaftlichen Regionalöko-

auf der Basis linearer Regression (Töpfer, 1973), die funktionale Typisierung von Städten (Wienke, 1973) und Stadtquartieren (Braun, 1975), die formale Netzwerktheorie (Leusmann, 1974) und geographische Modellvorstellungen (Mittelstädt, 1974) sowie die innerstädtische Unternehmenskonzentration (Glebe, 1975). 
Tabelle 1. Artikel mit quantitativ-theoretischer Richtung in der "Zeitschrift für Wirtschaftsgeographie“ 1969 bis 1980.

\begin{tabular}{lrrrrrrr}
\hline & $1969 / 70$ & $1971 / 72$ & $1973 / 74$ & $1975 / 76$ & $1977 / 78$ & $1979 / 80$ & Summe \\
\hline Theorie & 0 & 2 & 2 & 0 & 2 & 2 & 8 \\
Methoden & 0 & 0 & 0 & 1 & 1 & 0 & 2 \\
Planung/Regionalpolitik & 2 & 0 & 0 & 6 & 2 & 3 & 13 \\
Fallstudien & 0 & 2 & 2 & 3 & 4 & 5 & 16 \\
\hline Summe & 2 & 4 & 4 & 10 & 9 & 10 & 39 \\
\hline
\end{tabular}

nomie (Lösch, 1944; v. Böventer, 1962) zusammengefasst und zunächst in der US-amerikanischen Wirtschaftsgeographie rezipiert wurden (Hoover, 1948). Ebenso kann man hier auf die „Regional Science“ verweisen, die sich in den USA in den 1950er Jahren als interdisziplinär arbeitender Studiengang hatte etablieren können (Isard, 1956). Beide Diskussionslinien beinhalteten auch das zweite Element, eine enge Anlehnung an die volkswirtschaftliche Neoklassik und an eine rationale und atomistische Handlungstheorie, sowie das dritte Element, eine neopositivistische Methodologie. Letzteres verbreitete sich dann in der „Quantitativen Revolution“ der angelsächsischen Humangeographie und führte ab der Mitte der 1960er Jahren zu substanziellen Forschungserträgen (Chorley und Haggett, 1967; Berry und Marble, 1968; Harvey, 1969).

Möchte man nun den RA im deutschsprachigen Kontext situieren, genügt es dann, ihn als eine Adaption der oben dargestellten Theorieelemente zu kennzeichnen?

\section{Raumdeterminismus, Untersozialisierung und Quantifizierung?}

Die in Abschnitt 3 erwähnten drei Charakteristika, die dem RA zugeschrieben werden, lassen sich bei dessen Vertretern nur mit deutlichen Divergenzen identifizieren. Das erste Kriterium einer chorologischen Erklärungsstrategie scheint vorderhand gerechtfertigt zu sein durch die prominente Stellung der transportkostenbasierten Ansätze im akademischen Lehrgebäude. Allerdings hat bereits Schätzl (1974:125) bei der ersten Vorstellung des RA vermutet, dass die nichtlagebasierten Erklärungsfaktoren, namentlich die verschiedenen Varianten von internen und externen Ersparnissen, im Zeitablauf an Bedeutung gewinnen könnten. Im Laufe der Jahre nahm dann die Gewichtung organisationaler und institutioneller Faktoren in der Lehrbuchdarstellung zu (vgl. Schätzl, 1998:205). Wissen und Innovation in ihrer Rolle für den raumwirtschaftlichen Strukturwandel avancierten zu den bevorzugten Forschungsgegenständen (z.B. Fritsch et al., 1998). Sie stellen bei mehreren Habilitationen der zweiten und dritten Generation der Hannoveraner Schule die relevanten Erklärungsfaktoren dar (z.B. Sternberg, 1998; Koschatzky, 2001; Revilla Diez, 2002; Liefner, 2006). Auch in der Lehrbuchdarstellung hat sich mit den Organisations- und Netzwerktheorien ein neuer Theoriekomplex etabliert, der inzwischen qualitativ und quantitativ dominiert (Liefner und Schätzl, 2012).

Gegen das zweite Kriterium, die Anlehnung an die neoklassische Wirtschaftstheorie inklusive den Verhaltensannahmen zum atomistischen, rationalen und informierten Akteur spricht, dass bereits zu Beginn und seitdem zunehmend Theorien mit gegenläufigen Verhaltensannahmen und Wirkungszusammenhängen dargestellt wurden. Dies galt zuerst für die Polarisationstheorie und die (post-)keynesianische Wachstumstheorie (Schätzl, 1978:100 ff.), bei deren Schilderung etwa die unvollkommene Information als Verhaltensannahme eingeführt wird, der Staat als gleichberechtigter Akteur auftritt und sich ein längerfristig ungleichgewichtiges Wachstum ereignen kann. Ähnliche Abweichungen ergaben sich mit der intensiven Rezeption der New Economic Geography (Sternberg, 2000). All dies spricht dagegen, den RA als Theorie aufzufassen, der z.B. spezifische Verhaltensannahmen vertritt. So wird der RA von seinen Vertretern explizit als Agenda der gesamten Wirtschaftsgeographie eingeführt, d.h. mit der Aufgabe ,räumliche Strukturen und ihre Veränderungen [...] zu erklären, zu beschreiben und zu bewerten“" (Schätzl, 1978:16., Liefner und Schätzl, 2012:12). Demnach war das Ziel des RA ,nicht [...] eine $>$ neue $<$ Wirtschaftsgeographie zu kreieren [...], [sondern] die Wettbewerbsfähigkeit der geographischen Wissenschaft gegenüber Nachbardisziplinen auch in Zukunft zu erhalten“ (Schätzl, 1978:7).

Zum dritten Kriterium, der Ausrichtung auf den (Neo-)Positivismus, kann gesagt werden, dass eine wissenschaftstheoretische Grundlegung durch Bartels (1968a) als ausreichend angesehen wurde. Die Methodologie besteht in der Forderung nach empirischen Tests von Theorien (Schätzl, 2003:143), womit eine Nähe zur Hypothesenbildung und zum Falsifikationsverfahren des kritischen Rationalismus auf der Hand liegt. Allerdings sind Mathematisierung und Quantifizierung nicht an sich konstitutiv für den Ansatz. Qualitative Verfahren der empirischen Sozialforschung wurden von Anfang an mitberücksichtigt und Habilitationen können auch mit Dokumentenanalysen und Experteninterviews bestritten werden (z.B. Kiese, 2012). Umgekehrt hatte bereits Giese (1980:256) darauf aufmerksam gemacht, dass quantitative Verfahren nicht alleine vom RA, sondern auch von anderen Ansätzen verwendet würden. Inzwischen ist die Beherrschung von mathematisch- 
statistischen Verfahren überhaupt keine differentia specifica mehr, mit der etwa institutionalistische, politökonomische oder evolutorische Ansätze vom raumwirtschaftlichen Ansatz unterschieden werden könnten.

Kurzum, die heute gängige Darstellung des RA greift zu kurz, wenn sie diesen vor allem über die Merkmale Raumdeterminismus, Untersozialisierung und Quantifizierung kennzeichnet. Durch diese Eingrenzungen werden die interne Heterogenität und Wandlungsfähigkeit dieses Ansatzes nicht abgebildet. In welcher Hinsicht existiert dann überhaupt ein raumwirtschaftlicher Ansatz?

\section{Die Konstitution einer Expertenkultur}

Der RA stellte eine Innovation innerhalb der wirtschaftsgeographischen Disziplin dar, weil er sich über einen neuen Verwendungszusammenhang für das disziplinäre Wissen konstitutierte. Nachdem sich die ursprünglichen Adressaten der idiographisch-länderkundlichen Geographie aus der Gründungszeit des späten 19. Jhds., d.h. kommerzielle und militärische Informationsbedürfnisse und die Pflege des National- und Heimatbewußtseins, verflüchtigt hatten, bediente die Geographie in den 1960er Jahren nurmehr ein diffuses Bildungsbedürfnis in einer kleiner werdenden Welt. Daneben entstand jedoch das Bedürfnis an wissenschaftlich fundierter Beratung, Begründung und Evaluation raumbezogener Vorhaben privater und öffentlicher Akteure (Bartels, 1968b:128). Der RA orientierte sich mit seinen Modellen und Methoden auf diesem neuen Verwendungszusammenhang praktischer Akteure, und zwar insbesondere auf staatliche Administrationen auf verschiedenen Raumebenen. In diesem Sinne spricht Ludwig Schätzl (1974:131) in seiner Gießener Antrittsvorlesung von der „Aufgabe, einen Beitrag zur Lenkung des räumlichen Prozessablaufs in Richtung auf eine Optimierung wirtschafts- und gesellschaftspolitischer Zielsetzungen zu leisten“. In seinem für den RA grundlegenden Lehrbuch „Wirtschaftsgeographie 1. Theorie“ wird die normative Orientierung „Entscheidungshilfen für die Regionalpolitik“ zu geben bekräftigt (Schätzl, 1978:17 f., 2003:143, vgl. Liefner und Schätzl, 2012:17; vgl. Hauser und Rambousek, 1980 als zeitgenössischen Kommentar). Sie erst verband bereits in der ersten Ausgabe des Lehrbuchs die Theorien unterschiedlicher Provenienz zu Standort, Wachstum und Mobilität. In den folgenden Ausgaben wurden zusätzlich die Industriedistrikt-Ansätze, die marxistische Regulationstheorie oder die Netzwerktheorie adaptiert. Der Fluchtpunkt des RA ist somit die gesellschaftliche Verwertung; die „bewusste Gestaltung ökonomischer Raumsysteme" soll wissenschaftlich unterstützt werden mit allen verfügbaren wissenschaftlichen Erkenntnissen bzw. Instrumenten (Schätzl, 1986:16). Die Verbindung von Theorieund Methodenelementen im RA erschließt sich folglich über diesen unbedingten Praxisbezug: Welche Probleme sollten gelöst werden, wer sind die Adressaten der wissenschaftli- chen Expertise, welche Themen- und Methodenwahl ist damit verbunden? Dieser Fragenkomplex soll im Folgenden zumindest grob strukturiert werden.

Die Herausbildung einer planungsbezogenen Expertenkultur war eine disziplinübergreifende Entwicklung in den Sozialwissenschaften, in der sich wesentliche gesellschaftliche Strukturveränderungen nach dem Zweiten Weltkrieg reflektierten (Raphael, 1996; Brückweh et al., 2012). Unter dem Eindruck eines starken Wirtschaftswachstums und des fordistischen Klassenkompromisses entstand in den westlichen Industrieländern ein hoher Planungsbedarf, z.B. in den Bereichen Verkehr, Stadt, Industrie und Daseinsvorsorge. Die generelle Antwort bestand in einem technokratischen, entideologisierten Ausbau der staatlichen Planung und Steuerung, wirtschaftspolitisch wurde die keynesianische Globalsteuerung zur dominierenden Strategie. Dabei wurde aufgrund verschiedener Aspekte auch ein Raumbezug der Planung relevant, aus dem ein umfassender gesellschaftspolitischer Auftrag abgleitet wurde (Leendertz, 2008 und 2012; Etzemüller, 2012). Erstens wurde die Lebensführung de-lokalisiert bzw. auf neuen räumlichen Maßstabsebenen organisiert. So wurden z.B. im Bildungssystem die Zwergschulen geschlossen und im kulturellen System neue Bibliotheken und Musikschulen mit weiten Einzugsradien gegründet. Im ländlichen Raum verringerte sich die Erwerbstätigkeit im Agrarsektor und urbane Lebensformen hielten Einzug (Erker, 1996; Münkel, 2000). Die Politik z.B. in der Bundesrepublik Deutschland antwortete hierauf mit dem Zentrale-Orte-Programm ab 1957 und mit der Kreis- und Gebietsreform von 1967 bis 1978. Zweitens sollten regionale Disparitäten mit öffentlichen Investitionen (z.B. Universitätsgründungen) und insbesondere mit den neuen Instrumenten der regionalen Strukturpolitik, die ab 1968 als Bund-Länder-Gemeinschaftsaufgabe implementiert wurden, ausgeglichen werden. Drittens verstärkte sich ein raumbezogener Planungsbedarf in der internationalen Dimension, z.B. bei der Planung grenzüberschreitender Verkehrswege und in der seit den 1960er Jahren entstehenden Zusammenarbeit mit Entwicklungsländern.

Die Wirtschaftswissenschaften wurden zudem zum Vorbild im Adressatenwechsel, da sie sich in der Bundesrepublik bereits seit der Mitte der 1950er Jahre auf die Steuerbarkeit von Wirtschaft und Gesellschaft, auf die Verwissenschaftlichung der Politik und auf eine aktive staatliche Konjunkturund Wachstumspolitik orientiert hatten (Nützenadel, 2005; Scharnetzky, 2007). Hierzu wurden neue statistische, ökonometrische und prognostische Methoden eingeführt sowie neue Beratungsgremien eingerichtet, in denen Wissenschaft, Politik und Wirtschaft zusammentrafen.

Angesichts dieser bereits ausgeprägten Forschungslandschaft bestand der Innovationsgehalt des RA in der deutschsprachigen Wirtschaftsgeographie zunächst in einer intensiven Rezeption der oben erwähnten Diskussionslinien der Regionalökonomie und der „Regional Science“ und in einer inhaltlichen und methodischen Verbesserung der akade- 
mischen Ausbildungsinhalte. Da sich der Adressatenwechsel in Deutschland aber erst ab dem Jahr 1980 als nachholende Modernisierung auf breiter Front in Lehre und in den Qualifizierungsarbeiten durchsetzen konnte, war man damit konfrontiert, dass die genannten Akteure, insbesondere Wirtschaftswissenschaftler, in den neu entstandenen Planungsstäben, Programmbüros und wissenschaftlichen Beiräten bereits fest verankert waren. Tatsächlichen forderte die Wirtschaftsgeographie ihr Stück vom Beratungskuchen zu einer Zeit ein, als die Planungseuphorie längst verflogen und die finanziellen Spielräume längst wieder geschrumpft waren (vgl. Giese, 1980:259). Trotzdem konnte die Wirtschaftsgeographie an der raumbezogenen Planung partizipieren, z.B. mit Beratungsleistungen zu Technologie- und Gründerzentren, zur Wirkung öffentlicher Einrichtungen, zur Regionalisierung der Strukturpolitik in den 1980ern Jahren und der Clusterförderung in den 1990er Jahren.

Dieser gesellschaftliche Wandel führte auch zu wissenschafts- und bildungssysteminternen Veränderungen, die seitens der Wirtschaftsgeographie bewältigt werden mussten. Mit der Reform der Lehrerbildung und der gymnasialen Oberstufe zu Beginn der 1970er Jahre wurde auch durch die Bildungsbürokratie eine stärkere Verwissenschaftlichung des Schulunterrichts und der Lehrerbildung gefordert. Die damit verbundenen Forderungen nach gesellschaftlicher Relevanz und Reflexionsfähigkeit des Faches und die Sorgen um sinkende Arbeitsmarktchancen bildeten auch den Kern der studentischen Kritik auf dem Kieler Geographentag (Fachschaften der Geographischen Institute, 1970:193). Die Länderkunde wurde von den Studierenden pragmatisch als Hindernis bei einer thematischen und methodischen Modernisierung gesehen, zumal sie „mit $40 \%$ aller Veranstaltungen eine einsame Vorrangstellung" einnahm (ebd. 195 f.). In dieser Konstellation konnte das Fach zum einen von dem Ausbau der universitären und gymnasialen Kapazitäten in der zweiten Hälfte der 1960er Jahre profitieren, indem etwa spezialisierte Professuren für Wirtschaftsgeographie eingerichtet wurden. Zum anderen wurden seit der Mitte der 1960er Jahre durch den Aufbau von Diplom-Studiengängen Ausbildungskapazitäten geschaffen, mit denen Studierende für außerschulische Praxisfelder qualifiziert werden konnten. Bemerkenswert ist zudem, dass es in der Besetzungspolitik zu einer temporären Öffnung gegenüber wirtschaftswissenschaftlich ausgebildeten Fachvertretern kam und inneruniversitär organisatorische Verbindungen von Wirtschaftsgeographie und Wirtschaftswissenschaft eingegangen wurden (Schamp, 2007:240 f.; Schätzl, 2003:143 f.). Diese Veränderungen vergrößerten die Chancen auf einen Generationenwechsel, innerhalb dessen der RA seinen Einfluss ausdehnen konnte.

Mit der Umdefinition der Wirtschaftsgeographie zu einer Fachkultur von beratenden Experten nahm der RA sowohl den gesellschaftlichen Trend einer Neujustierung von Wissenschaft und Politik auf, als auch das innerfachliche Bedürfnis von Studenten und Dozenten an einer nachhal- tigen Berufsperspektive. Das Verdienst des RA ist es, die Erschließung dieses Verwendungskontextes für die Subdisziplin Wirtschaftsgeographie offensiv vorangetrieben zu haben.

\section{Die Normativität von „Praxis“}

Der Bezug der Wirtschaftsgeographie auf ,gesellschaftliche Relevanz", „Verwertbarkeit" und "Praxis" hat zum einen etwas heutzutage Selbstverständliches. Auf diesem, vom RA wesentlich zugänglich gemachten Terrain stehen auch gegenwärtige wirtschaftsgeographische Strömungen, die sich auf ein ,gesellschaftszentriertes Raumkonzept“ (Krätke, 1995:5 ff.) oder eine ,sozialtheoretische Revolution“ (Bathelt und Glückler, 2012:31 ff) berufen. Mit der Abkehr vom idiographisch-länderkundlichen Paradigma wechselte in der Wirtschaftsgeographie das Verhältnis von Theorie, Empirie und Anwendungsbezug des Wissens. Die Verwendbarkeit wirtschaftsgeographischen Wissens innerhalb von gesellschaftlichen Handlungskontexten wurde zu einem Prüfkriterium der theoretischen Entwicklung und machte eine Pluralität von konkurrierenden Ansätzen bzw. ein sozialwissenschaftliches Standardniveau erst möglich. Nun konnte der Theorie-Korpus über empirische Tests, Theorievergleiche und begriffliche Arbeit verändert werden, während zuvor die konzeptionellen Diskussionen regelmäßig im ästhetisierten Begriff der „Landschaft“" erstickt worden waren (Bartels, 1969).

Zum anderen hat der RA den Bezug zur Praxis in einer spezifischen Weise hergestellt, die das Feld der Theorie- und Methodenentwicklung in der deutschsprachigen Wirtschaftsgeographie stark geprägt hat. Erstens, der RA grenzte den möglichen Verwendungszusammenhang von wirtschaftsgeographischem Wissen vor allem auf die „bewußte Gestaltung ökonomischer Raumsysteme durch den Staat bzw. öffentliche Institutionen“ ein (Schätzl, 1986:16; kritisch hierzu: Schamp, 1983:74). Zunächst wurde eine Liaison mit dem keynesianischen Wohlfahrtsstaat eingegangen, für den man eine „Analyse von Verlauf, Ursachen und Wirkung von Disparitäten in der regionalen Wirtschaftsentwicklung" betrieb (Schätzl, 1974:128; vgl. Schätzl, 1986:21 f.). Für den RA war somit die (administrative) Region die wesentliche räumliche Bezugsgröße und weniger der einzelwirtschaftliche Standort.

In den 1990ern wurden dann die inhaltlichen Koordinaten des RA in Richtung auf Marktkonformität, Innovation und Wettbewerbsfähigkeit verschoben. Damit blieb die Fokussierung auf ein politisch hegemoniales Projekt (LissabonStrategie) und auf die darauf verpflichtete staatliche Forschungsförderung bestehen. Diesem Aufmerksamkeitskegel entgingen jedoch wesentliche Problemfelder wie die Ökologisierung, die Bedeutung sozialer Milieus, der Aufstieg der Kreativwirtschaft oder der Konflikte in den industriellen Beziehungen. Diese relevanten Praxiszugänge wurden von institutionalistischen, polit-ökonomischen und kultura- 
listischen Ansätzen in der Wirtschaftsgeographie hergestellt. Die Auseinandersetzung über die Bedeutung dieser Ansätze wird vom RA zweitens über materielle (Drittmittel, Qualifikationsarbeiten) und immaterielle Erfolgskriterien (z.B. Rankings) geführt (vgl. die Urteile über verschiedene Theoriekonzepte in Schätzl, 2003:142). Hier kann gefragt werden, ob eine wünschenswerte Qualitätssicherung und Profilbildung der Disziplin nicht eine zu starke Oberhand über die Geltung ,guter Argumente“ in einer wissenschaftlichen Community erhalten. Langfristig kann sich diese Betonung von Input- und Output-Erfolgsmassen auch $\mathrm{zu}$ einem $\mathrm{Bu}-$ merang für den RA erweisen, da die mit ihm konkurrierenden Ansätze von neuen Akteuren in den genannten Praxiszugängen profitieren. So werden wirtschaftsgeographische Projekte inzwischen in nennenswertem Maße über gesellschaftspolitisch engagierte Stiftungen, über politische Parteien, Umweltverbände oder Gewerkschaften finanziert. Zudem schlägt sich die Pluralisierung der gesellschaftlichen Interessen in den staatlichen Instanzen selbst nieder, und zwar auf allen Raumebenen von der EU-Forschungsförderung bis hin zu regionalen und kommunalen Einrichtungen. Dies lässt die Konkurrenz um Praxiszugänge auch zu einem Kampf um die Interpretation wissenschaftlicher Ansätze innerhalb von staatlichen Institutionen werden, was den dritten Zugang des RA zur Praxis begründet, seine Formierung als soziales Phänomen. Als strukturierter Ausbildungsweg, Karrierenetzwerk und Zitationsgemeinschaft kann die „Hannoveraner Schule“ an den Vergabestellen staatlicher Mittel effizient Einfluss ausüben. Gerade weil der RA nicht mit einem inhaltlichen Aussagekern identifizierbar ist, kann und muss er seine materiellen Ressourcen engagiert sichern.

Kurzum, der „Praxisbezug“ wurde vom RA zunächst als Nachweis für die gesellschaftliche Legitimität der gesamten Disziplin Wirtschaftsgeographie eingeführt und der RA verstand sich als neutraler Problemlöser extern vorgegebener Probleme. Durch die spezifische Ausrichtung des RA auf (hegemoniale) politische Projekte hat er jedoch nur einen Teil der gesellschaftlichen Problemfelder bearbeitet. Die inzwischen auch im deutschsprachigen Raum beheimateten alternativen theoretischen Ansätze bearbeiten diese lange ignorierten Problemfelder und haben sich eigene Zugänge zu gesellschaftlichen Akteuren und materiellen Ressourcen geschaffen.

\section{Fazit: Essentials für eine Historisierung des raumwirtschaftlichen Ansatzes}

Das Ziel dieses Beitrages war es, die Rolle des RA in der heutigen Diskussion über die theoretischen und thematischen Ausrichtungen der Wirtschaftsgeographie neu zu justieren. Hierzu können sechs Essentials festgehalten werden.

Erstens, soweit man theoriehistorisch an der Abkehr von der idiographisch-länderkundlichen (Wirtschafts-)Geographie interessiert ist, ist es sinnvoll sich länderübergreifend auf die Entwicklung zur „Spatial Analysis" in den USA und in Großbritannien seit dem Beginn der 1960er Jahre und disziplinübergreifend auf die Entstehung der Standort- und Raumwirtschaftstheorien in der volkswirtschaftlichen Regionalökonomie und in der „Regional Science“ seit dem Ende der 1940er Jahre zu beziehen. Sofern man zweitens an einer auf den deutschsprachigen Raum bezogenen Fachgeschichte interessiert ist, stellen die methodologischen Innovationen von Dietrich Bartels und die Auseinandersetzungen auf dem Kieler Geographentag 1969 wichtige Bezugspunkte dar, die aber als punktuelle und somit symbolische Konflikte einzuordnen sind. Erst in einer nachholenden Modernisierung ab der zweiten Hälfte der 1970er Jahre setzten sich ein neues Fachverständnis, neue Lehrinhalte und veränderte Forschungsmethoden durch, wobei der RA als ein wichtiger Impulsgeber aufzufassen ist.

Drittens, in theoriesystematischer Perspektive war der RA keine bloße Fortsetzung der „Spatial Analysis“ und kann nicht auf Theorieelemente wie die Kausalität physischer Lagedistanzen, das neoklassische Wirtschaftsmodell sowie auf eine neopositivistische Methodologie reduziert werden. Vielmehr bildete er ein Set aus heterogenen Theorie- und Methodenelementen, die durch den gemeinsamen Anwendungsbezug einer beratungsorientierten Expertenkultur zusammengehalten wurden. Eine ausschließlich auf die wissenschaftstheoretischen Grundlagen und die theoretischen Konzepte bezogene Kritik könnte somit eher an die ,Spatial Analysis“" gerichtet werden, würde jedoch den RA wenig treffen. In dessen Zentrum steht das Streben nach der Sicherung materieller Ressourcen in Form von Stellen- und Sachmitteletats für die Subdisziplin Wirtschaftsgeographie. Viertens, mit dem Selbstverständnis der Wirtschaftsgeographie als einer problemlösenden Sozialwissenschaft wurde der gesellschaftliche Wandel der Nachkriegsgesellschaft mit ihrem Anstieg an Planungsbedarfen aufgenommen. Da das Komplexitätsniveau westlicher Gesellschaften seitdem dauerhaft hoch geblieben ist, hat sich diese Nachfrage nach raumbezogener Kompetenz zwar in den Zielen und Instrumenten verändert, aber grosso modo verstetigt. Fünftens hat der RA diesen Anwendungsbezug spezifisch ausgestaltet, in dem man sich sehr eng an die Instanzen staatlicher Steuerung und Forschungsförderung anlehnte. Der Themen- und Theoriewechsel des RA vom Ausgleich regionaler Disparitäten bis zur Gestaltung innovationsorientierter Netzwerk- und Clustereinrichtungen ist somit die Geschichte der politisch hegemonialen Projekte. Sechstens, die gegenwärtig relevanten Strömungen der institutionellen, kulturalistischen und politökonomischen Wirtschaftsgeographie unterscheiden sich vom RA zum einen durch ihren jeweiligen Theoriekern. Damit bezieht sich ihr Wettbewerb nicht ausschließlich auf die Kriterien und Rankings des materiellen Inputs und Outputs. Zum anderen adressieren sie alternative Anwendungsbezüge wirtschaftsgeographischen Wissens im ökologischen Umbau, in der Qualität von Arbeitsplätzen oder gegenüber dem domi- 
nant gewordenen Finanzsektor, die dem RA bislang als randständig erschienen sind.

Damit ist die Gegenwart des pluralen Methoden- und Theorienspektrums in der Wirtschaftsgeographie erreicht: Gerade weil die heutigen fachlichen Ansätze in ihrer großen Mehrheit (auch) dem Postulat des Anwendungsbezugs folgen, ist das Streben des RA nach einer Vereinheitlichung von Theorien und Methoden (vgl. Sternberg, 2000) in weite Ferne gerückt. Die gesellschaftlichen Konfliktlinien haben zu einer Ausdifferenzierung auch innerhalb der Subdisziplin geführt. Die Orientierung auf scheinbar neutrale Steuerungsansätze ist einer Diskussion konkurrierender gesellschaftspolitischer Entwürfe gewichen. Eine zukünftige Theoriegeschichtsschreibung der Wirtschaftsgeographie kann dies als Entfaltung des sozialwissenschaftlichen Paradigmas beschreiben: das wirtschaftsgeographische Wissen entwickelt sich so divers wie seine gesellschaftlichen Anwendungsbezüge.

Daten-Verfügbarkeit. Es wurden keine Datensätze für diesen Artikel genutzt.

Interessenkonflikt. Der Autor erklärt, dass kein Interessenkonflikt besteht.

Danksagung. Ich bedanke mich bei den beiden anonymen Reviewern für ihre konstruktiven Hinweise.

Edited by: B. Korf

Reviewed by: two anonymous referees

\section{Literatur}

Arnreiter, G. und Weichhart, P.: Rivalisierende Paradigmen im Fach Geographie, in: Koexistenz rivalisierender Paradigmen. Eine post-kuhnsche Bestandsaufnahme zur Struktur gegenwärtiger Wissenschaft, Herausgeber: Schurz, G. und Weingartner, P., Westdeutscher Verlag, Opladen, 1998.

Bahrenberg, G.: Von der Anthropogeographie zur Regionalforschung - eine Zwischenbilanz, in: Zur Situation der deutschen Geographie zehn Jahre nach Kiel, Herausgeber: Sedlacek, P., Selbstverlag, Osnabrück, 59-68, 1979.

Bahrenberg, G.: Paradigmenwechsel in der Geographie: Vom Regionalismus über den raumwissenschaftlichen Ansatz wohin?, in: Geographie und Gesellschaftstheorie. Beiträge zur Bevölkerungs- und Sozialgeographie 3, Herausgeber: Matznetter, W., Institut für Geographie der Universität Wien, Wien, 2532, 1995.

Bahrenberg, G.: Die Länderkunde im Paradigmenstreit um 1970, Berichte zur deutschen Landeskunde 70, 41-54, 1996.

Barnes, T. J.: Probable Writing: Derrida, Deconstruction, and The Quantitative Revolution in Human Geography, Environ. Plann. A, 26, 1021-1040, 1994.
Barnes, T. J.: Logics of Dislocation. Models, Metaphors, and Meanings of Economic Space, Guilford, New York, 1996.

Barnes, T. J.: Envisioning Economic Geography: Three Men and their Figures, Geog. Z., 86, 94-105, 1998.

Barnes, T. J.: "In the beginning was economic geography" - a science studies approach to disciplinary, Prog. Hum. Geogr., 25 , 521-544, 2001.

Barnes, T. J.: The place of locational analysis: a selective and interpretive history, Prog. Hum. Geogr., 27, 69-95, 2003.

Barnes, T. J.: Lost in translation: Wirtschaftsgeographie als ,trading zone", in: Denkanstöße zu einer anderen Geographie der Ökonomie, Herausgeber: Berndt, Ch. and Glückler, J., Transcript, Bielefeld, 25-46, 2006.

Bartels, D.: Zur wissenschaftstheoretischen Grundlegung einer Geographie des Menschen, Steiner, Wiesbaden, 1968a.

Bartels, D.: Die Zukunft der Geographie als Problem ihrer Standortbestimmung, Geog. Z., 56, 124-142, 1968b.

Bartels, D.: Der Harmoniebegriff in der Geographie, Die Erde, 100, 124-137, 1969.

Bartels, D.: Leitbilder der Raumordnung als quantifizierte Zuordnungsmodelle, Z. f. Wirtgeo., 14, 65-79, 1970.

Bartels, D.: Die konservative Umarmung der „Revolution“. Zu Eugen Wirths Versuch in „Theoretischer Geographie“, Geog. Z., 68, 121-131, 1980.

Bathelt, H. und Glückler, J.: Wirtschaftsgeographie. Ökonomische Beziehungen in räumlicher Perspektive. 3. Aufl., Ulmer, Stuttgart, 2012.

Beck, H.: Die Gebietsreform in der Bundesrepublik Deutschland, Z. f. Wirtgeo., 19, 197-200, 1975.

Belina, B., Best, U., and Naumann, M.: Critical geography in Germany: from exclusion to inclusion via internationalization, Soc. Geogr., 4, 47-58, 2009.

Berry, B. J. L. und Marble, D. F.: Spatial Analysis. A Reader in Statistical Geography, Prentice Hall, Englewood Cliffs, NJ, 1968.

Blotevogel, H. H.: „Neue Kulturgeographie“ - Entwicklung, Dimensionen, Potenziale und Risiken einer kulturalistischen $\mathrm{Hu}-$ mangeographie, Berichte zur deutschen Landeskunde, 77, 7-34, 2003.

Böventer, E. v.: Theorie des räumlichen Gleichgewichts, Mohr, Tübingen, 1962.

Braun, A.: Homogene Stadteinheiten: Probleme ihrer Ausgliederung und Qualifizierung, Z. f. Wirtgeo., 19, 97-103, 1975.

Braun, B. und Schulz, C.: Wirtschaftsgeographie, Ulmer, Stuttgart, 2012.

Brückweh, K., Schumann, D., Wetzell, R. F., and Ziemann, B. (Hrsg.): Engineering Society. The Role of the Human and Social Sciences in Modern Societies, 1880-1980, Palgrave Macmillan, Basingstoke, 2012.

Burton, I.: The Quantitative Revolution and Theoretical Geography, Can. Geogr., 7, 151-162, 1963.

Chorley, R. und Haggett, P.: Models in Geography, Methuen, London, 1967.

Cox, K. R.: Making human geography, Guilford Press, New York, 2014.

Cresswell, T.: Geographic thought: a critical introduction, WileyBlackwell, Chichester u.a.O., 2013.

Dorner, H.: Wirtschaftsgeographie, Bohmann, Wien, 1975.

Eisel, U.: Die Entwicklung der Anthropogeographie von einer „Raumwissenschaft“ zur Gesellschaftswissenschaft. Urbs et Re- 
gio, Kasseler Schriften zur Geografie und Planung, Band 17, Gesamthochschul-Bibliothek, Kassel, 1980.

Elsasser, H.: Das Investitionshilfegesetz für Berggebiete, Z. f. Wirtgeo., 19, 185-186, 1975.

Erker, P.: Der lange Abschied vom Agrarland. Zur Sozialgeschichte der Bauern im Industrialisierungsprozeß, in: Politische Zäsuren und gesellschaftlicher Wandel im 20. Jahrhunderts. Regionale und vergleichende Perspektiven, Forschungen zur Regionalgeschichte 18, Herausgeber: Frese, M. and Prinz, M., Ferdinand Schöningh, Paderborn, 327-360, 1996.

Etzemüller, T.: Strukturierter Raum - integrierte Gemeinschaft. Auf den Spuren des social engineering im Europa des 20. Jahrhunderts, in: Theorien und Experimente der Moderne. Europas Gesellschaften im 20. Jahrhundert, Herausgeber: Raphael, L., Böhlau Verlag, Köln, Weimar, Wien, 129-154, 2012.

Fachschaften der Geographischen Institute: Bestandsaufnahme zur Situation der deutschen Schul- und Hochschulgeographie, Herausgeber: Meckelein, W. und Borcherdt, C., Tagungsbericht und wissenschaftliche Abhandlungen. Deutscher Geographentag Kiel, 21.-26. Juli 1969. Verhandlungen des deutschen Geographentages 37, Steiner, Wiesbaden, 191-207, 1970,

Fritsch, M., Koschatzky, K., Schätzl, L., and Sternberg, R.: Regionale Innovationspotentiale und innovative Netzwerke - Zum Stand der Forschung, Raumforschung und Raumordnung, 56, 243-252, 1998.

Gaebe, W.: Grundlagen und Bestrebungen zu einer Neugliederung des Bundesgebietes, Z. f. Wirtgeo., 14, 97-108, 1970.

Giese, E.: Entwicklung und Forschungsstand der „Quantitativen Geographie“ im deutschsprachigen Bereich, Geog. Z., 68, 256283, 1980.

Glebe, G.: Beobachtungen zur Konzentration ausländischer Firmen in der Innenstadt Düsseldorfs, Z. f. Wirtgeo., 19, 20-24, 1975.

Gregory, D.: Alfred Weber and Location Theory, in: Geography, Ideology and Social Concern, Herausgeber: Stoddart, D. R., Basil Blackwell, Oxford, 165-185, 1981.

Gregory, D.: Geographical imaginations, Blackwell, Cambridge, MA und Oxford, 1994.

Haas, H.-D.: Strukturelle Merkmale neugegründeter und verlagerter Industrie im Nordosten Baden-Württembergs, Z. f. Wirtgeo., 15, 246-250, 1971.

Haas, H.-D. und Neumair, S.-M.: Wirtschaftsgeographie, Wissenschaftliche Buchgesellschaft, Darmstadt, 2007.

Hard, G.: Die Disziplin der Weißwäscher. Über Genese und Funktion des Opportunismus in der Geographie, in: Osnabrücker Studien zur Geographie, 2, 11-44, 1979.

Hard, G. und Fleige, H.: Quantitatives zur ,quantitativen und theoretischen Revolution“ in der deutschsprachigen Geographie: Zitierverhalten in Methodologie, Stadtgeographie und Geographiedidaktik 1945-73: Eine Studie zur Stabilität und Veränderung des geographischen Informationshorizontes seit 1945, Karlsruher Manuskripte zur Mathematischen und Theoretischen Wirtschafts- und Sozialgeographie, Geographisches Institut, Karlsruhe, 1978.

Harvey, D. W.: Explanation in Geography, Arnold, London, 1969.

Hauser, C. and Rambousek, W. H.: Der raumwirtschaftliche Ansatz in der Geographie, Geogr. Helv., 35, 89-92, doi:10.5194/gh-3589-1980, 1980.

Hoover, E.: The Location of Economic Activity, Mc-Graw-Hill, New York, 1948.
Isard, W.: Location and Space Economy, John Wiley \& Sons, New York, 1956.

Kiese, M.: Regionale Clusterpolitik in Deutschland. Bestandsaufnahme und interregionaler Vergleich im Spannungsfeld von Theorie und Praxis, Metropolis Verlag, Marburg, 2012.

Kilchenmann, A.: Zum gegenwärtigen Stand der Quantitativen und Theoretischen Geographie. Karlsruher Manuskripte zur Mathematischen und Theoretischen Wirtschafts- und Sozialgeographie 1, Geographisches Institut, Karlsruhe, 1975.

Knoblauch, H.: Wissenssoziologie. Konstanz, UVK Verlagsgesellschaft, 2005.

Koschatzky, K.: Räumliche Aspekte im Innovationsprozess. Ein Beitrag zur neuen Wirtschaftsgeographie aus Sicht der regionalen Innovationsforschung, Wirtschaftsgeographie 19, LIT Verlag, Münster, 2001.

Krätke, S.: Stadt, Raum, Ökonomie. Einführung in aktuelle Problemfelder der Stadtökonomie und Wirtschaftsgeographie, Birkhäuser, Basel, Boston und Berlin, 1995.

Krumbein, W., von Frieling, H.-D., Kröcher, U., and Sträter, D. (Hrsg.): Kritische Regionalwissenschaft: Gesellschaft, Politik, Raum, Verlag Westfälisches Dampfboot, Münster, 2008.

Krumme, G.: Anmerkungen zur Relevanz unternehmerischer Verhaltensweisen in der Industriegeographie, Z. f. Wirtgeo., 16, 101-108, 1972.

Kulke, E.: Wirtschaftsgeographie. 3. Aufl., Schöningh, München, Wien und Zürich, 2008.

Läpple, D.: Essay über den Raum. Für ein gesellschaftswissenschaftliches Raumkonzept, in: Stadt und Raum: soziologische Aspekte, Herausgeber: Häußermann, H. u.a., Stadt, Raum und Gesellschaft 1, Centaurus-Verlag, Pfaffenweiler, 157-207, 1991.

Leendertz, A.: Ordnung schaffen. Deutsche Raumplanung im 20. Jahrhundert, Wallstein-Verlag, Göttingen, 2008.

Leendertz, A.: Das Soziale Ordnen. Sozialwissenschaften und gesellschaftliche Ungleichheit im 20. Jahrhundert, Eigene und fremde Welten 27, Herausgeber: Reinecke, C. und Mergel, T., Campus Verlag, Frankfurt, New York, 337-369, 2012.

Leusmann, C.: Zur Gliederung von Netzen, Z. f. Wirtgeo., 18, 239247, 1974.

Lichtenberger, E.: Klassische und theoretisch-quantitative Geographie im deutschen Sprachraum, Ber. z. Raumforschung u. Raumplanung, 22, 9-20, 1978.

Liefner, I.: Ausländische Direktinvestitionen und internationaler Wissenstransfer nach China - untersucht am Beispiel von Hightech-Unternehmen in Shanghai und Beijing, Wirtschaftsgeographie 34, LIT Verlag, Münster, 2006.

Liefner, I. und Schätzl, L.: Theorien der Wirtschaftsgeographie. 10. Aufl., Schöningh, Wien, Köln und Weimar, 2012.

Lösch, A.: Die räumliche Ordnung der Wirtschaft, 2. Aufl., Gustav Fischer, Jena, 1944.

Maier, G. und Tödtling, F.: Regional- und Stadtökonomik 1. Standorttheorie und Raumstruktur. 4. Aufl., Springer, Wien und New York, 2006.

Michel, B.: Wir sind nie revolutionär gewesen - Zum Mythos des Kieler Geographentags als der Geburtsstunde einer neuen Geographie, Geogr. Helv., 69, 301-303, doi:10.5194/gh-69-3012014, 2014.

Mittelstädt, G.: Modellvorstellungen in der Geographie, Z. f. Wirtgeo., 18, 45-49, 1974. 
Morikawa, H.: Entwicklungstypen der zentralörtlichen Hierarchie in Japan, Z. f. Wirtgeo., 15, 48-59, 1971.

Moulines, C. U.: Die Entwicklung der modernen Wissenschaftstheorie (1890-2000). Eine historische Einführung, LIT Verlag, Hamburg, 2008.

Münkel, D. (Hrsg.): Der lange Abschied vom Agrarland. Agrarpolitik, Landwirtschaft und ländliche Gesellschaft zwischen Weimar und Bonn, Göttingen, 2000.

Nickel, A. und Piepenstock, O.: Wirtschaftsgeographie. Teil I: Deutschland. 15. Aufl., Winkler, Darmstadt, 1972.

Nützenadel, A.: Stunde der Ökonomen. Wissenschaft, Politik und Expertenkultur in der Bundesrepublik 1949-1974, Vandenhoeck und Ruprecht, Göttingen, 2005.

Otremba, E.: Die Wirtschaft der Erde im Wandel, 7., überarb. Aufl., Gehlen, Bad Homburg vor d. Höhe, Berlin, Zürich, 1978.

[o.V.]: Das Emsland in der regionalen Strukturpolitik der Bundesregierung, Z. f. Wirtgeo., 15, 157-158, 1971.

Peet, R.: Modern Geographical Thought, Blackwell, Oxford, 1998.

Raphael, L.: Die Verwissenschaftlichung des Sozialen als methodische und konzeptionelle Herausforderung für eine Sozialgeschichte des 20. Jahrhunderts, Gesch. Ges., 22, 165-193, 1996.

Reidel, F., Uhl, E. und Westphal, E.: Land und Leute gestalten die Wirtschaft: eine Wirtschaftsgeographie, 70. Aufl., Winkler, Darmstadt, 1973.

Revilla Diez, J.: Betrieblicher Innovationserfolg und räumliche Nähe: zur Bedeutung innovativer Kooperationsverflechtungen in metropolitanen Verdichtungsregionen; die Beispiele Barcelona, Stockholm und Wien, Wirtschaftsgeographie 22, LIT Verlag, Münster, 2002.

Schamp, E. W.: Grundansätze der zeitgenössischen Wirtschaftsgeographie, Geographische Rundschau, 3, 74-80, 1983.

Schamp, E. W.: Denkstile in der deutschen Wirtschaftsgeographie, Z. f. Wirtgeo. 51, 238-252, 2007.

Schätzl, L.: Zur Konzeption der Wirtschaftsgeographie, Die Erde, 105, 124-134, 1974.

Schätzl, L.: Wirtschaftsgeographie 1. Theorie. Schöningh, Paderborn, 1978 .

Schätzl, L.: Wirtschaftsgeographie 3, Politik. Schöningh, Paderborn, 1986.

Schätzl, L.: Wirtschaftsgeographie 1. Theorie. 7. Aufl., Schöningh, Paderborn, 1998.

Schätzl, L.: Zur Zukunft der Wirtschaftsgeographie in Deutschland, Z. f. Wirtgeo. 47, 139-144, 2003.

Scharnetzky, T.: Die große Ernüchterung. Wirtschaftspolitik, Expertise und Gesellschaft in der Bundesrepublik 1966 bis 1982, Akademie-Verlag, Berlin, 2007.

Schöller, P. und Liedtke, H.: Tagungsbericht und wissenschaftliche Abhandlungen. Deutscher Geographentag Erlangen-Nürnberg, 1.-4. Juni 1971. Verhandlungen des deutschen Geographentages Bd. 38. Steiner, Wiesbaden, 1972.

Schultz, H.: Die deutschsprachige Geographie von 1800 bis 1970. Ein Beitrag zur Geschichte ihrer Methodologie, Selbstverlag des Geographischen Instituts der Freien Universität Berlin, Berlin, 1980.
Scott, A. J.: Economic Geography: The Great Half-Century, in: The Oxford Handbook of Economic Geography, Herausgeber: Clark, G. L., Feldman, M. P. und Gertler, M. S., Oxford, 18-44, 2000.

Sedlacek, P.: Zur Situation der deutschen Geographie zehn Jahre nach Kiel, Selbstverlag, Osnabrück, 1979.

Sheppard, E.: Quantitative geography: Representations, practices, and possibilities, Environ. Plann. D, 19, 535-554, 2001.

Sheppard, E.: We have never been positivist, Urban Geogr., 35, 636-644, 2014.

Siebert, A.: Raumordnung in Europa, Z. f. Wirtgeo., 7, 133-143, 1963.

Sternberg, R.: Technologiepolitik und High-Tech-Regionen - ein internationaler Vergleich, Wirtschaftsgeographie 7, LIT Verlag, Münster, 2. veränd. Aufl., 1998.

Sternberg, R.: Stand, Selbstverständnis und Perspektiven der deutschen Wirtschaftsgeographie, in: Würzburger Geographische Arbeiten 94, 33-57, 2000.

Töpfer, H.: Befragung von Geschäftsinhabern zur Erfassung der Einzelhandelsstruktur, Z. f. Wirtgeo., 17, 1-6, 1973.

von Frieling, H.-D.: Raum und Regionalentwicklung in der neoklassischen Ökonomie - ein kritischer Überblick, in: Kritische Regionalwissenschaft: Gesellschaft, Politik, Raum, Herausgeber.: Krumbein, W., von Frieling, H.-D., Kröcher, U., and Sträter, D., Verlag Westfälisches Dampfboot, Münster, 168-190, 2008.

Voppel, G.: Wirtschaftsgeographie. Schaeffers Grundriß des Rechts und der Wirtschaft 98. Kohlhammer, Stuttgart und Düsseldorf, 1970.

Wagner, H.: Wirtschaftsgeographie. Westermann, Braunschweig, 1981.

Weichhart, P.: Paradigmenvielfalt in der Humangeographie - Neue Unübersichtlichkeit oder Multiperspektivität?, in: Fachdidaktik: alternativ - innovativ. Acht Impulse um (Schul-)Geographie und ihre Fachdidaktik neu zu denken, Herausgeber: Vielhaber, Ch.: Materialien zur Didaktik der Geographie und Wirtschaftskunde 17, Selbstverlag des Instituts für Geographie und Regionalforschung der Universität Wien, Wien, 11-19, 2004.

Weichhart, P.: Der Kieler Geographentag 1969 - Modernisierungsschub, Mythos, Paradigmenwandel oder vergessene Geschichte?, Geogr. Helv., 71, 7-13, doi:10.5194/gh-71-7-2016, 2016.

Wenzler, J.: Allgemeine Wirtschaftsgeographie, 14. Aufl., Winkler, Darmstadt, 1972.

Wienke, H. M.: Citystruktur und ihre Ursachen, Z. f. Wirtgeo., 17, 149-157, 1973.

Wirth, E.: Zum Problem einer allgemeinen Kulturgeographie, Die Erde, 100, 161-193, 1969.

Winkler, E.: Landesplanerische Leitbilder der Schweiz, Z. f. Wirtgeo., 19, 65-69, 1975.

Wirth, E.: Zur wissenschaftstheoretischen Problematik der Länderkunde, Geog. Z., 66, 241-261, 1978.

Wirth, E.: Theoretische Geographie, Teubner, Stuttgart, 1979. 